Research Article

\title{
Experimental Study on the Transport of Light Gas Molecules through Low-Density Polyethylene Films
}

\author{
Riccardo Checchetto \\ Dipartimento di Fisica, Università di Trento, via Sommarive 14, Povo, 38123 Trento, Italy \\ Correspondence should be addressed to Riccardo Checchetto; riccardo.checchetto@unitn.it
}

Received 10 July 2018; Accepted 30 August 2018; Published 25 October 2018

Academic Editor: Miriam H. Rafailovich

Copyright ( 2018 Riccardo Checchetto. This is an open access article distributed under the Creative Commons Attribution License, which permits unrestricted use, distribution, and reproduction in any medium, provided the original work is properly cited.

\begin{abstract}
An original experimental procedure for the study of gas permeation process through thin polymer films is presented. Employing mass spectroscopy techniques, this procedure allows the detection of the permeation flux with a signal-to-noise ratio large enough to obtain accurate measurements of the gas diffusivity also in processes with transient transport conditions lasting for short-interval times ( few seconds). The procedure is validated using as test material a thin low-density polyethylene (LDPE) film: the transport of four test gases with different molecular sizes and condensation properties $\left(\mathrm{CO}_{2}, \mathrm{~N}_{2}, \mathrm{D}_{2}\right.$, and $\left.\mathrm{He}\right)$ is studied in the 295 to $350 \mathrm{~K}$ temperature interval. The $\mathrm{CO}_{2}$ diffusivity values well compare with values previously obtained studying the same LDPE film samples by integral permeation technique measuring the time lag value. Original data on the diffusivity of the $\mathrm{He}$ and $\mathrm{D}_{2}$ penetrant molecules are reported: in the examined temperature range, the diffusivity values of these small-size penetrants are in the $10^{-6} \mathrm{~cm}^{2} / \mathrm{s}$ range and follow an Arrhenius behavior with temperature. The activation energy values for diffusion are $18.8 \pm 0.4$ and $10.0 \pm 0.4 \mathrm{~kJ} / \mathrm{mol}$ for $\mathrm{D}_{2}$ and $\mathrm{He}$, respectively.
\end{abstract}

\section{Introduction}

The study of mass transport process through polymer films is an important research area given its applicative interest for the development of innovative membranes for gas separation [1] and of gas barrier films to be used in the packaging technology [2]. This topic also presents fundamental interest: gas diffusion occurs by successive jumps of the penetrant molecules between cavities formed in the polymer matrix by the random thermal fluctuations of the macromolecular chains $[3,4]$. These cavities constitute the free volume of the polymer which controls structural properties such as the glass transition temperature [5]. Penetrant molecules thus act as sensitive probe to explore the polymer-free volume and its variation, for example, with temperature and cross-linking density $[6,7]$ or with the addition of filler nanoparticles [8], and to analyze the structure of rigid cavities in biopolymer membranes [9].

The gas transport through polymer membranes obeys to the solution-diffusion mechanism [1] which assumes as transport parameters the molecular diffusivity $D$ (units: $\mathrm{cm}^{2} / \mathrm{s}$ ) and solubility $\Pi$ (units: $\mathrm{mol} \cdot \mathrm{cm}^{-3} \cdot \mathrm{Pa}^{-1}$ ) in the polymer layers (the gas permeability $\Phi$ is given by the product of gas solubility and diffusivity, $\Phi=D S$ ). Measurements are carried out by gas-phase permeation technique often using planar membrane samples shaped in the form of a thin disc with a diameter much larger than the disc thickness $L$. Keeping the sample temperature $T$ constant, at time $t=0$ (which is the trigger time of the experiment), one side of the planar membrane is exposed to the test gas kept at fixed pressure $P_{\text {HPS }}$. This side of the membrane is called the high-pressure side (HPS). The other side is called the lowpressure side (LPS) and faces an evacuated analysis chamber kept in vacuum conditions. The gas permeation process consists in the transfer of gas molecules from the HPS of the membrane to the analysis chamber through the membrane layers and occurs in three microscopic steps:

(i) Absorption: penetrant molecules in the gas phase are absorbed by the HPS surface layers of the membrane. Here, their concentration immediately reaches its equilibrium value $c_{\text {HPS }}=c(x=0, t)=\Pi$ $P_{\text {HPS. Here, } x=0}$ represents the coordinate of the HPS 
(ii) Diffusion step: molecules absorbed in the HPS of the membrane diffuse through the membrane layers to the LPS down to their concentration gradient according to the first Fick law, $j(x, t)=-D(\partial c(x, t) / \partial x)$; $c(x, t)$ is the gas concentration at time $t$ in the membrane layers at depth $x$ below the HPS

(iii) Desorption step: molecules reaching the LPS surface layers of the membrane desorb in the analysis chamber. When $P_{\text {LPS }}^{\text {gas }} \ll P_{\mathrm{HPS}}$, the approximation $c_{\mathrm{LPS}}=c$ $(x=L, t)=\Pi P_{\text {LPS }}^{\text {gas }} \cong 0$ holds $(x=L$ is the coordinate of the LPS, that is, the membrane thickness)

The permeation flux $j(t)=j(x=L, t)=-D(\partial c(x=L, t) /$ $\partial x)$ can be obtained solving Fick's second equation $\partial c(x, t) / \partial t=D\left(\partial^{2} c(x, t) / \partial x^{2}\right)$.

The previous diffusion problem admits for $j(t)$ the following analytical solution:

$$
j(t)=\frac{D \Pi}{L} P_{\mathrm{HPS}}\left[1+2 \sum_{n=1}^{\infty}(-1)^{n} \exp \frac{-D n^{2} \pi^{2} t}{L^{2}}\right] .
$$

Steady-state transport conditions are set when $j(t)$ assumes the constant, time-stationary value $J=(D \Pi / L) P_{\text {HPS }}$.

Gas molecules permeate through the membrane of surface area $A$ at a rate $Q(t)=A j(t)$. Molecules permeated in the analysis chamber form a rarefied gas at thermal equilibrium with the chamber walls at temperature $T_{\text {cham }}$.

The partial pressure $P_{\text {LPS }}^{\text {gas }}(t)$ of the test gas varies, as a function of time, according to the following relation:

$$
Q(t)=\frac{1}{R T_{\text {cham }}}\left[V \frac{d P_{\mathrm{LPS}}^{\mathrm{gas}}(t)}{d t}+S_{p} P_{\mathrm{LPS}}^{\mathrm{gas}}(t)\right],
$$

where $R$ is the universal gas constant, $V$ is the volume of the analysis chamber, and $S_{p}$ is the pumping speed of the vacuum system.

During the permeation experiments, the pressure $P_{\exp }(t)$ in the analysis chamber is measured as a function of time: the analysis of the $P_{\exp }(t)$ curve by (2) permits to measure the permeation flux $j(t)$ and to obtain the values of the $D$ and $\Phi$ transport parameters.

Gas-phase permeation experiments are carried out by integral methods $[10,11]$ : at $t=0$, the pumping system is excluded (i.e., $S_{p}=0$ ) and permeated molecules accumulate in the analysis chamber. According to the solutiondiffusion model, at time $t$, the total number of permeated moles $m(t)$ is given by [12]

$$
\begin{aligned}
m(t)= & A \int_{t^{\prime}=0}^{t^{\prime}=t} j\left(t^{\prime}\right) d t^{\prime}=A\left\{\frac{D \Pi P_{\mathrm{HPS}}}{L}\left[t-\frac{L^{2}}{6 D}\right]\right. \\
& \left.-2 \frac{L \Pi P_{\mathrm{HPS}}}{\pi^{2}} \sum_{n=1}^{\infty} \frac{(-1)^{n}}{n^{2}} \exp \frac{-D n^{2} \pi^{2} t}{L^{2}}\right\},
\end{aligned}
$$

and the partial pressure of the test gas in the analysis chamber $P_{\text {LPS }}^{\text {gas }}(t)$ increases as a function of time as $P_{\text {LPS }}^{\text {gas }}(t)=$ $\left(R T_{\text {cham }} / V\right) m(t)$.

In the integral permeation experiments, the pressure in the analysis chamber can be recorded by using low-pressure gauges (in fact, $S_{p}=0$ and low-vacuum conditions are rapidly set). Gas transport parameters are obtained by the analysis of the experimental permeation curves $P_{\exp }(t)$ when steadystate transport conditions are set, that is, when the $P_{\exp }(t)$ value linearly increases with time $t$.

According to the model, in steady state conditions $j(t)=J=(D \Pi / L) P_{\mathrm{HPS}}$, thus, $m(t)=A\left(D \Pi P_{\mathrm{HPS}} / L\right)\left[t-\left(L^{2} /\right.\right.$ $6 D)]$ and

$$
P_{\mathrm{LPS}}^{\mathrm{gas}}(t)=\frac{R T_{\text {cham }}}{V} \frac{A}{L} D S P_{\mathrm{HPS}}\left[t-\frac{L^{2}}{6 D}\right] .
$$

The measured rate of pressure increase $d P_{\exp }(t) / d t$ provides the gas permeability $\Phi=\Pi D$ by the relation $d P_{\text {exp }}(t) / d t=d P_{\text {LPS }}^{\text {gas }}(t) / d t=\left(R T_{\text {cham }} / V\right)(A / L) D S P_{\text {HPS }}$. The intercept of the straight line fitting the measured pressure data with the time axis, according to (4), defines a parameter called time lag given by $\tau_{\text {lag }}=L^{2} / 6 D$ : this characteristic time is a measure of the lasting of transient transport conditions and permits the evaluation of the gas diffusivity $D$.

The accuracy of this procedure in the evaluation of the gas transport parameter is limited by the background flow $Q_{\text {back }}$ due to degassing from the chamber walls $\left(\mathrm{H}_{2} \mathrm{O}\right.$ and $\mathrm{CO}$ adsorbed during the venting procedure), to small vacuum leaks $\left(\mathrm{H}_{2} \mathrm{O}\right.$ vapor, $\mathrm{N}_{2}$, and $\left.\mathrm{O}_{2}\right)$, and to air permeation through Viton O-rings $\left(\mathrm{H}_{2} \mathrm{O}\right.$ vapor, $\mathrm{N}_{2}$, and $\left.\mathrm{O}_{2}\right)$. During the permeation test, $Q_{\text {back }}$ increases the pressure $P_{\text {LPS }}^{\text {back }}(t)$ of the gases forming the residual vacuum: the pressure $P_{\text {LPS }}^{\text {tot }}(t)$ measured in the analysis chamber is thus given by two contributions $P_{\text {exp }}(t)=P_{\text {LPS }}^{\text {back }}(t)+P_{\text {LPS }}^{\text {gas }}(t)$ which cannot be resolved by the total pressure gauge.

For a given sample, the accuracy in the evaluation of the gas permeability $\Phi$ rises by improving the signal-to-noise ratio $S / N=\left(d P_{\mathrm{LPS}}^{\mathrm{gas}} / d t\right) /\left(d P_{\mathrm{LPS}}^{\mathrm{back}}(t) / d t\right)$, that is decreasing the sample thickness $L$. The $P_{\text {LPS }}^{\text {back }}(t)$ term affects the accuracy in the measure of $P_{\mathrm{LPS}}^{\mathrm{gas}}(t)$ and introduces large relative indetermination in the evaluation of the $\tau_{\text {lag }}$ parameter which impedes to obtain accurate measures of the gas diffusivity studying the fast transport process as it occurs with thin film samples or analyzing the transport of penetrants with high $D$ values or membrane samples made of low-permeability materials [13-16]. Note also that in permeation processes with penetrant diffusivities $D$ in the $10^{-6} \mathrm{~cm}^{2} / \mathrm{s}$ order, using film samples with thickness $L$ in the $10 \mu \mathrm{m}$ range, time lag values are in the few-second order in which the result is thus comparable to that of the response time of the low-pressure gauges.

In this paper, we present an original experimental procedure for the study of the gas permeation process which permits accurate evaluation of the gas diffusivity (and permeability) of thin polymer films and processes characterized by 


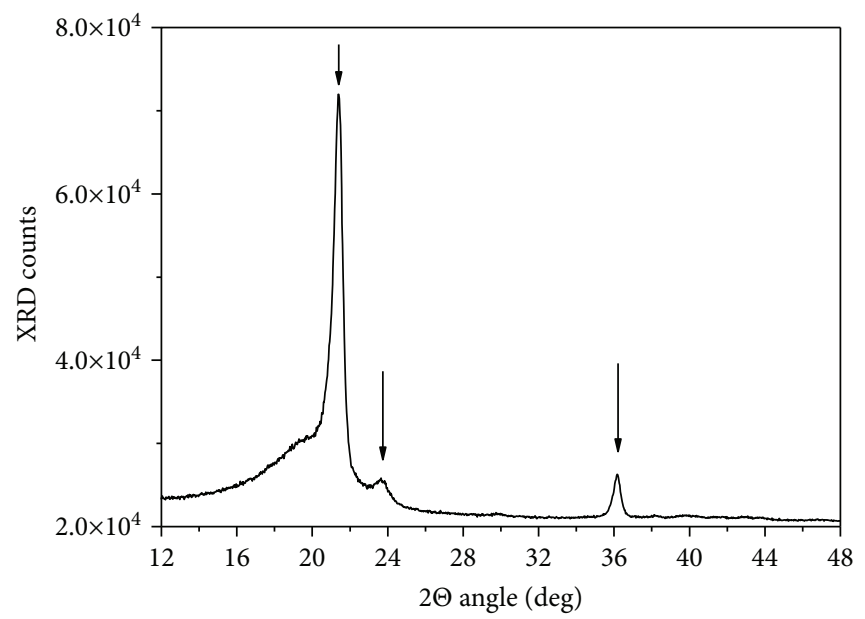

FIGURE 1: XRD spectrum of the LDPE film ( $C u K_{\alpha}$ radiation, $\lambda=1.5414 \AA$ ). The arrow indicates the LDPE reflection peaks.

short transient times: this often occurs in the permeation of small-size penetrants and in high-temperature processes. The validation of the proposed experimental approach will be carried out using thin films made of low-density polyethylene (LDPE) which is a polymer used as gas barrier in packaging applications [17].

Measurements were carried out using test gases with different molecular sizes, molecular masses, and condensation properties and analyzing the transport process up to temperatures close to the LDPE melting temperature. Our measurements permitted us to obtain original data on the diffusivity of the light and small-size $\mathrm{D}_{2}$ and $\mathrm{He}$ penetrant molecules, and analysis of the diffusivities permitted the evaluation of the $\mathrm{D}_{2}$ and $\mathrm{He}$ activation energy for diffusion which are original data here presented.

\section{Experimental Part}

The study was carried out using membrane samples made of commercial semicrystalline low-density polyethylene (LDPE) kindly provided by ICBM-CNR (Naples, Italy). The X-ray diffraction pattern (XRD) of the LDPE films is presented in Figure 1. The spectrum shows sharp, narrow diffraction peaks due to the crystalline fraction of the material at $2 \theta=21.1^{\circ}, 2 \theta=23.8^{\circ}$, and $2 \theta=36^{\circ}$ (see vertical arrows) while the amorphous component gives a very broad peak. The Bragg-Brentano analysis of the XRD peak at $2 \theta=21.1^{\circ}$ indicated that the average size of PE crystallites is about $12 \mathrm{~nm}$. The crystalline fraction of the LDPE samples, as evaluated by DSC analysis, was $29 \pm 1 \%$. The melting temperature $T_{m}$ of the present LDPE samples was $\sim 380 \mathrm{~K}$ [18].

The study of the gas transport properties of the present samples was previously carried out by the procedure described in the introduction, and measurements were carried out in the 290 to $350 \mathrm{~K}$ temperature range using $\mathrm{H}_{2}$, $\mathrm{CO}_{2}$, and $\mathrm{N}_{2}$ [18]. Reliable measurements of the permeability and diffusivity values were possible only with the $\mathrm{CO}_{2}$ test gas. Diffusivity values ranged from $\sim 1 \times 10^{-7} \mathrm{~cm}^{2} / \mathrm{s}$ at $290 \mathrm{~K}$ to $\sim 1 \times 10^{-6} \mathrm{~cm}^{2} / \mathrm{s}$ at $350 \mathrm{~K}$ while the activation energy values for diffusion and permeation were $29.7 \pm 0.4 \mathrm{~kJ} / \mathrm{mol}$ and $36.4 \pm 0.4 \mathrm{~kJ} / \mathrm{mol}$, respectively. Using the $\mathrm{H}_{2}$ and $\mathrm{N}_{2}$ test gases, it was possible to evaluate only the gas permeability because of the large relative indetermination in the short time lag values [18].

In the procedure, we are here describing that the permeation experiment is carried out under continuous pumping of the analysis chamber which is done by turbomolecular or sputter-ion pumps: high vacuum conditions are thus maintained during the permeation test.

When the condition

$$
\frac{S_{p}}{V} \gg \frac{1}{P_{\mathrm{LPS}}^{\mathrm{gas}}(t)} \frac{d P_{\mathrm{LPS}}^{\mathrm{gas}}(t)}{d t},
$$

is satisfied, then according to (2), the permeation flux $j(t)$ is directly proportional to the instantaneous value, $P_{\text {LPS }}^{\text {gas }}(t)$, of the test gas partial pressure

$$
Q(t)=A j(t)=S_{p} P_{\mathrm{LPS}}^{\mathrm{gas}}(t)
$$

In the present vacuum system, $\left(S_{p} / V\right)>5 s^{-1}$ and the condition $\left(S_{p} / V\right) \gg\left(1 / P_{\text {LPS }}\right)\left(d P_{\text {LPS }}(t) / d t\right)$ are satisfied for each test gas in the examined temperature range.

The $P_{\text {LPS }}^{\text {gas }}(t)$ curves can be thus converted to experimental permeation flux curves $j_{\exp }(t)$ through the relation

$$
j_{\exp }(t)=\frac{1}{A} \frac{1}{R T_{\text {cham }}} S_{p} P_{\text {LPS }}^{\text {gas }}(t) .
$$

Following this procedure,

(i) the measured value of the permeation flux $J_{\exp }$ in stationary transport conditions permits the evaluation of the penetrant permeability $\Phi=D \Pi$ by the relation $J_{\text {exp }}=J=(D \Pi / L) P_{\mathrm{HPS}}$ 
(ii) the penetrant diffusivity $D$ can be obtained fitting the normalized $j_{\exp }(t) / J_{\exp }$ permeation curves with the curve $j_{\text {norm }}(t)=j(t) / J=1+2 \sum_{n=1}^{\infty}(-1)^{n} \exp \left(-D n^{2}\right.$ $\left.\pi^{2} t / L^{2}\right)$; see (1)

Let us discuss now the advantages of this "constantpumping" procedure. The continuous pumping of the analysis chamber during the experimental run maintains ultra high- (UHV) or high-vacuum (HV) conditions and, consequently, permits the use of a quadrupole mass spectrometer to detect the permeation signal $I_{\mathrm{LPS}}^{\text {gas }}(t)$, that is the ionic current pertinent to the test gas. Knowing the QMS sensitivity for the test gas, $\alpha_{\text {gas }}$, the $I_{\mathrm{LPS}}^{\text {gas }}(t)$ signal provides a measure of the partial pressure of the permeating gas, $P_{\mathrm{LPS}}^{\mathrm{gas}}(t)=I_{\mathrm{LPS}}^{\text {gas }} / \alpha_{\text {gas }}$. The $P_{\mathrm{LPS}}^{\text {gas }}(t)$ value is thus obtained, as a function of time, without noise contributions due to background flows of any other residual gas component.

This experimental procedure consequently allows

(i) very large the signal-to-noise ratio $S / N$. In fact, $S / N=P_{\text {LPS }}^{\text {gas }}(t) / \delta P_{\text {back }}^{\text {gas }}=I_{\text {LPS }}^{\text {gas }}(t) / \delta I_{\text {back }}^{\text {gas }}=$ where the noise $\delta P_{\text {back }}^{\text {gas }}=\alpha_{\text {gas }} \delta I_{\text {back }}^{\text {gas }}$ is due to the fluctuations of the background partial pressure $P_{\text {back }}^{\text {gas }}$ of the test gas, only. The $S / N$ is very large for all gases, in particular for those not forming the residual vacuum of the analysis chamber

(ii) very low detection limit of the permeation flux. For each test gas, in fact, the $S_{p} \delta P_{\text {back }}^{\text {gas }}=\alpha_{\text {gas }} S_{p} \delta$ $I_{\text {back }}^{\text {gas }}$ term defines the detection limit $(\mathrm{A} / \mathrm{Pa})$. Consequently, the permeation signal can be efficiently detected also studying using low-permeability materials and the permeation fluxes $j(t)$ can be detected also when $A j(t)<Q_{\text {back }}$

(iii) that the high sampling rate of the QMS, adjustable from $0.5 \mathrm{~ms} / \mathrm{amu}$ to $60 \mathrm{~s} / \mathrm{amu}$ (amu: atomic mass unit), permits to record $P_{\text {LPS }}^{\text {gas }}(t)$ data with high time resolution

To test the effectiveness of this "constant-pumping" (CP) approach and for its validation, we used disc-shaped, planar samples with $13.5 \pm 0.2 \mathrm{~mm}$ diameter and thickness $L=53 \pm 1 \mu \mathrm{m}$. Measurements were carried out in the 295 to $350 \mathrm{~K}$ temperature range with $P_{\mathrm{HPS}}$ values of $40 \mathrm{kPa}$ using test gas with different molecular sizes and condensation properties: carbon dioxide $\left(\mathrm{CO}_{2}\right)$, nitrogen $\left(\mathrm{N}_{2}\right)$, deuterium $\left(\mathrm{D}_{2}\right)$, and helium $(\mathrm{He})$; see Table 1 .

Experiments were carried out in a stainless steel analysis chamber pumped by turbomolecular pumps; the detection of the permeation signal is carried out by a QMS equipped with secondary electron multiplier (SEM) and grid-type ion source: this setup permits to detect partial pressures down to $10^{-12} \mathrm{~Pa}$.

A schematic diagram of the system is shown in Figure 2(a); for the sake of simplicity, in this figure, the tubular oven used for sample heating is not reported. In Figure 2(b), we present the results of the QMS calibration
TABLE 1: Chemical-physical properties of the examined test gases [19].

\begin{tabular}{lccc}
\hline Gas & $\begin{array}{c}\text { Critical temperature } \\
(\mathrm{K})\end{array}$ & $\begin{array}{c}\text { Kinetic diameter } \\
(\AA)\end{array}$ & $\begin{array}{c}\text { Molecular mass } \\
(\mathrm{amu})\end{array}$ \\
\hline $\mathrm{D}_{2}$ & 38.2 & 2.89 & 4 \\
$\mathrm{He}$ & 5.19 & 2.6 & 4 \\
$\mathrm{CO}_{2}$ & 304.2 & 3.3 & 44 \\
$\mathrm{~N}_{2}$ & 126.2 & 3.64 & 28 \\
\hline
\end{tabular}

procedure to evaluate the sensitivity $\alpha_{\text {gas }}$ for the examined test gases. Starting from background pressure levels $P_{\text {back }}$ lower than $10^{-6} \mathrm{~Pa}$, through a calibrated leak valve connected to the gas reservoir, the pressure of the test gas in the pumped analysis chamber is increased by a factor 10 and the corresponding QMS and IG signals were recorded. Repeating this procedure, couples of $\left[P_{L P S}(t) \operatorname{vs} L_{L P S}^{g a s}(t)\right]$ data were obtained and the slope of the line fitting them provides the value of the $\alpha_{\text {gas }}$ parameter. We obtained the following values $\alpha_{H e}=$ $125 \pm 1 \mathrm{~Pa} / \mathrm{A}, \alpha_{D 2}=210 \pm 1 \mathrm{~Pa} / \mathrm{A}, \alpha_{N 2}=342 \pm 3 \mathrm{~Pa} / \mathrm{A}$, and $\alpha_{\mathrm{CO} 2}=457 \pm 1 \mathrm{~Pa} / \mathrm{A}$.

In Figure 3(a), we present the mass spectrum of the background vacuum at $P_{\text {back }}=1.2 \times 10^{-6} \mathrm{~Pa}$, after one night of turbomolecular pumping: the spectrum reports the QMS mass signal $I_{\text {back }}^{\text {gas }}$ pertinent to the gases forming the residual vacuum. It can be seen that the background vacuum is mostly composed of water vapor $\left(\mathrm{H}_{2} \mathrm{O}^{+}, \mathrm{m} / \mathrm{e}=18\right.$, fragmentation products $\mathrm{OH}^{+}$at $\mathrm{m} / \mathrm{e}=17$, and $\mathrm{O}^{+}$at $\left.\mathrm{m} / \mathrm{e}=16\right)$, carbon monoxide $\left(\mathrm{CO}^{+}, \mathrm{m} / \mathrm{e}=28\right.$, fragmentation product $\mathrm{C}^{+}$at $\mathrm{m} / \mathrm{e}=12$ ), and molecular hydrogen $\left(\mathrm{H}_{2}{ }^{+}, \mathrm{m} / \mathrm{e}=2\right.$, fragmentation product $\mathrm{H}^{+}$at $\left.\mathrm{m} / \mathrm{e}=1\right)$ [19]. In Figure 3(b), we report the QMS mass signal for $\mathrm{N}_{2}$ (upper panel) and $\mathrm{D}_{2}$ (lower panel) recorded in the permeation test carried out at $295 \mathrm{~K}$ with the described "constant-pumping" procedure: vertical arrows indicate the trigger time $t=0$.

Looking at the curves for $t<0$, we observe that the QMS background signals $I_{\text {back }}^{\text {gas }}$ are in the $10^{-12}$ and $10^{-10} \mathrm{~A}$ order for $\mathrm{D}_{2}$ and $\mathrm{N}_{2}$ while the $\delta I_{\text {back }}^{\text {gas }}$ fluctuations are $\sim 2 \times 10^{-12} \mathrm{~A}$ for both gases: the $S / N$ is thus larger than 10 already in the initial instants of the permeation transient $(t>0)$ and reaches values in the $10^{3}$ order in stationary transport conditions.

The $\delta I_{\text {back }}^{\text {gas }}$ fluctuations in the $10^{-12} \mathrm{~A}$ order permit to evaluate the detection limit of the present experimental approach by the relation $\delta j \sim(1 / A)\left(1 / R T_{\text {cham }}\right) S_{p} \alpha_{\text {gas }} \delta I_{\text {back }}^{\text {gas }}$. Given the effective $S_{p}$ values in the $50 \mathrm{~L} / \mathrm{s}$ order, QMS sensitivity $\alpha_{\text {gas }} \sim 200 \mathrm{~Pa} / \mathrm{A}$, see Figure $1(\mathrm{~b})$, and membrane surface area $A \sim 1 \mathrm{~cm}^{2}$, then $\delta j$ is in the $10^{-14} \mathrm{~mol} \cdot \mathrm{cm}^{-2} \cdot \mathrm{s}^{-1}$ order $\left(T_{\text {cham }}=300 \mathrm{~K} ; R=8.3 \mathrm{~L} \cdot \mathrm{kPa} \cdot \mathrm{mol}^{-1} \mathrm{~K}^{-1}\right): \delta j$ well compare with the upper value of the oxygen transmission rate (OTR) for gas barrier films to be used in the organic light-emitting device (OLED) technology, $\sim 3 \times 10^{-14} \mathrm{~mol} \cdot \mathrm{cm}^{-2} \cdot \mathrm{s}^{-1}[20]$.

The permeation flux is obtained from the curves in Figure 3(a) subtracting the background QMS signal 


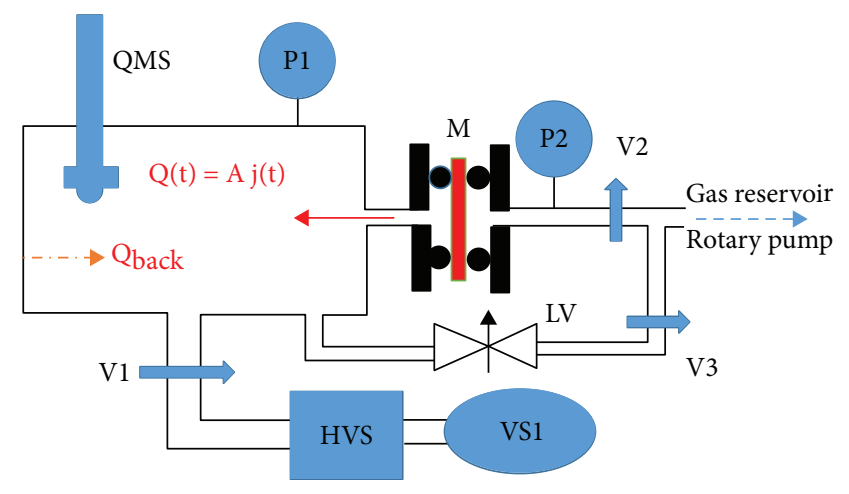

(a)

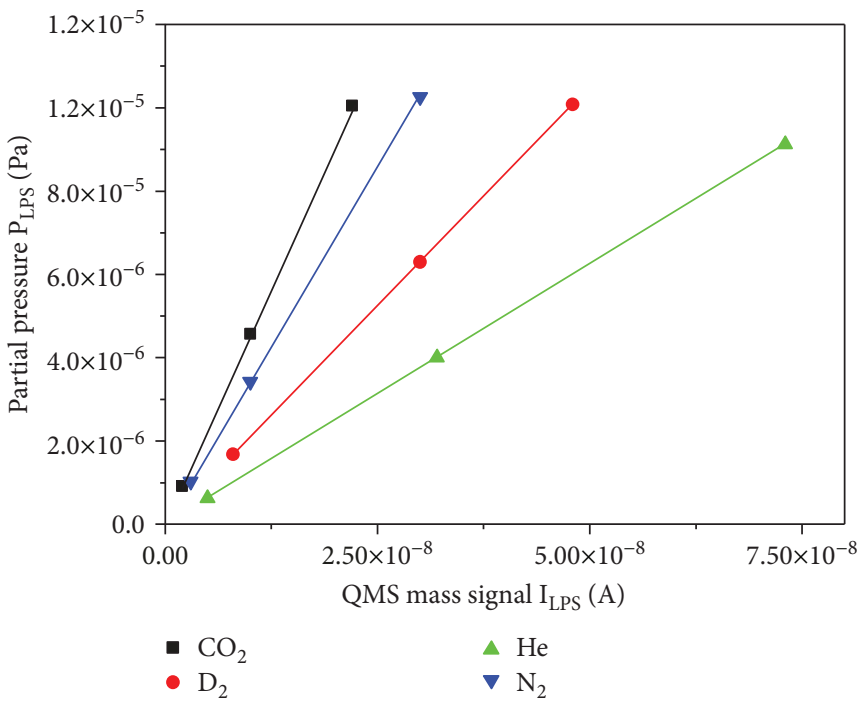

(b)

Figure 2: (a) Schematic of the experimental apparatus. QMS: quadrupole mass spectrometer; HVS: ultra high-vacuum pumping system; VS1: medium vacuum pumping system (diaphragm pump and molecular drag pump); P1: ionization gauge; P2: low-pressure gauge; V1, V2, and V3: vacuum valves; LV: calibrated leak valve. The membrane sample $(\mathrm{M})$ is vacuum sealed using two Viton O-rings. In this schematic diagram, the tubular oven for membrane sample heating is not reported. (b) QMS calibration curves. The QMS mass signal $I_{\text {LPS }}$ is reported as a function of the $P_{\text {LPS }}$ pressure value for the different test gases. Indetermination is inside the size of the symbol.

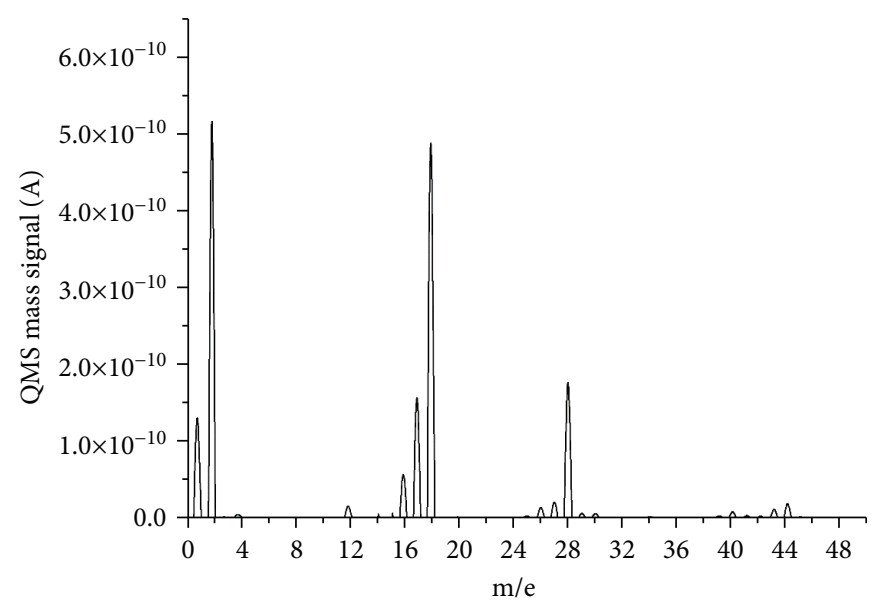

(a)

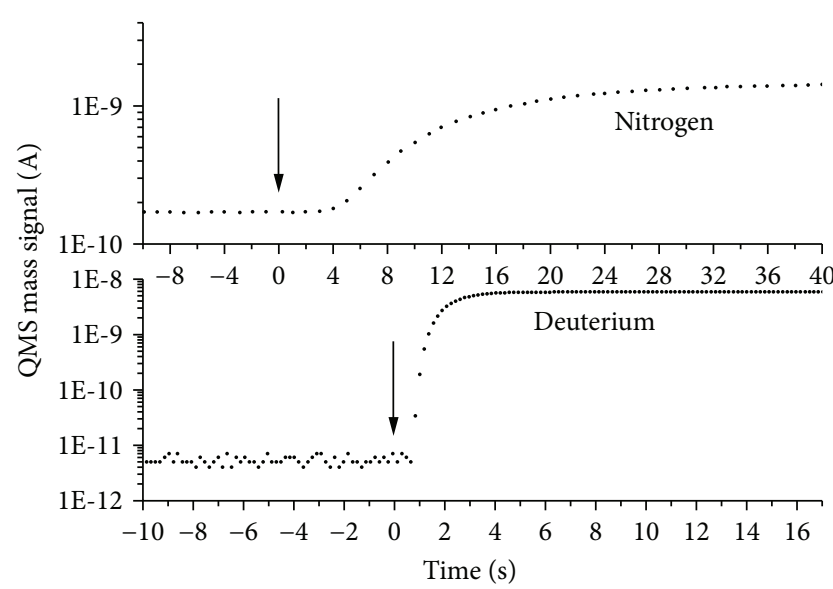

(b)

Figure 3: (a) Mass spectrum of the background vacuum at $P_{\text {back }}=1.2 \times 10^{-6} \mathrm{~Pa}$ after one night of turbomolecular pumping. The dominant contributions are related to the $I_{\text {back }}^{\text {gas }}$ signals pertinent to $\mathrm{H}_{2} \mathrm{O}, \mathrm{CO}$, and $\mathrm{H}_{2}(\mathrm{~m} / \mathrm{e}=18,28$, and 2, respectively). Their fragmentation products are also reported; see text. (b) QMS mass signals $I_{\mathrm{LPS}}^{D 2}$ and $I_{\mathrm{LPS}}^{N 2}(\mathrm{~m} / \mathrm{e}=4$ and 28 , respectively) recorded in the permeation test. Arrows indicate the trigger time of the experiment.

$I_{\text {back }}^{\text {gas }}$ from the detected QMS signal $I_{\text {LPS }}^{\text {gas }}(t)$ and using the relation

$$
j_{\text {exp }}(t)=\frac{1}{A} \frac{1}{R T_{\text {cham }}} S_{p} \alpha_{\text {gas }}\left[I_{\mathrm{LPS}}^{\text {gas }}(t)-I_{\text {back }}^{\text {gas }}\right]
$$

\section{Results and Discussion}

In Figure 4(a), we present the $j_{\exp }(t)$ curves as obtained at $295 \mathrm{~K}$ exposing the HPS at $40 \mathrm{kPa}$ pressure. In the figure, symbols are the experimental data (relative indeterminations are inside the size of the symbol) obtained by (8) while 


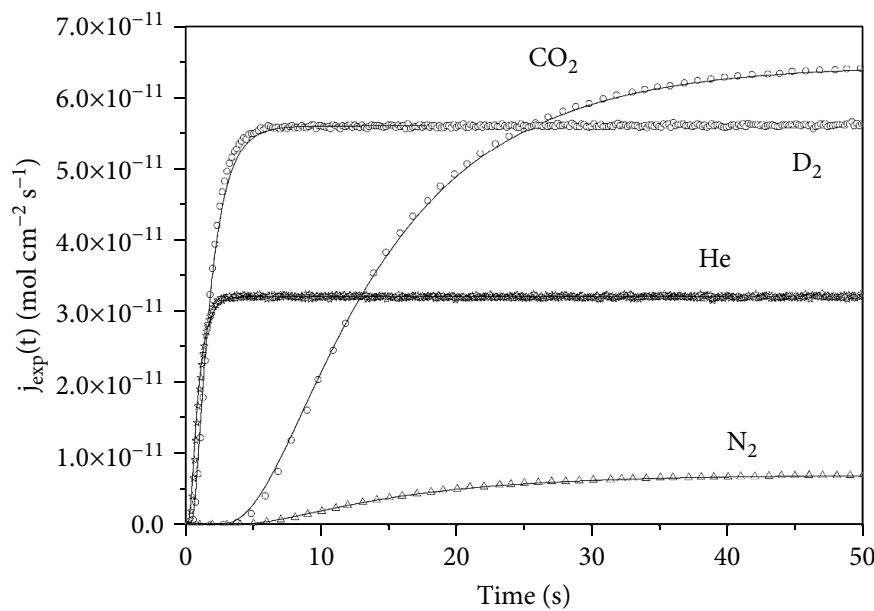

(a)

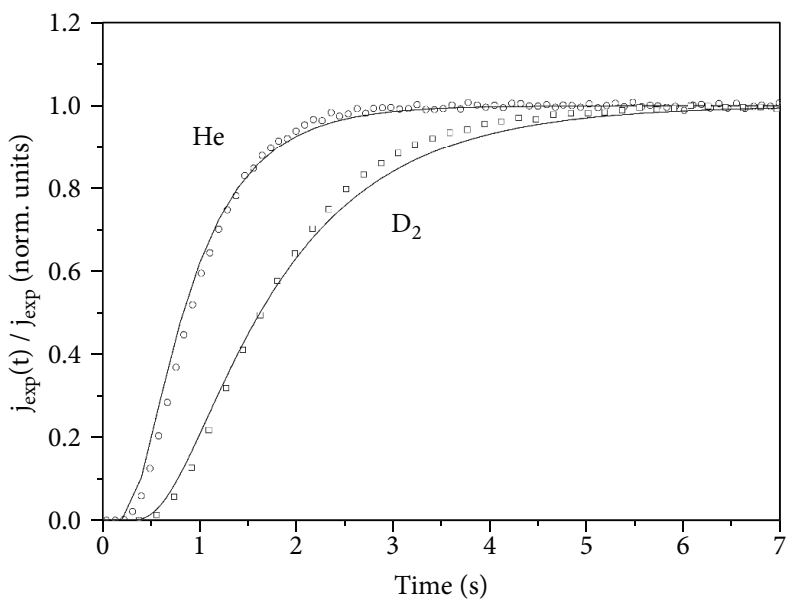

(b)

Figure 4: (a) Permeation flux curves $j_{\exp }(t)$ (open symbols) pertinent to $\mathrm{CO}_{2}, \mathrm{~N}_{2}, \mathrm{D}_{2}$, and $\mathrm{He}$ obtained at $T=295 \mathrm{~K}$ and $P_{\mathrm{HPS}}=40 \mathrm{kPa}$. Lines are fitting of the experimental curves; see text. (b) Normalized $j_{\exp }(t) / J_{\exp }$ curves (open symbols) pertinent to $\mathrm{D}_{2}$ and $\mathrm{He}$ obtained at $T=295 \mathrm{~K}$ and $P_{\mathrm{HPS}}=40 \mathrm{kPa}$. Lines are the $j_{\exp }(t) / J_{\text {exp }}$ curve fittings by the $j_{\text {norm }}(t)$ function; see text.

continuous lines are their numerical fit. All permeation curves are characterized by an initial interval time where penetrant occurs in transient conditions; the permeation flux then reaches a constant value indicating that stationary transport conditions are set. Fits were obtained first measuring the value of the permeation flux in stationary transport condition relation $J_{\exp }=(D \Pi / L) P_{\text {HPS }}$ to evaluate the gas permeability $\Phi$ $=D \Pi$ value and then fitting the normalized $j_{\exp }(t) / J_{\exp }$ curve with the $j_{\text {norm }}(t)$ function to evaluate the gas diffusivity $D$.

Comparing the curves in Figure 4(a), we observe that transient transport conditions last for longer interval times with the $\mathrm{CO}_{2}$ and $\mathrm{N}_{2}$ penetrants than with $\mathrm{D}_{2}$ and $\mathrm{He}$ which have smaller molecular size. The difference between the permeation curves in transient transport conditions pertinent to the small-size test molecules, namely, $\mathrm{D}_{2}$ and $\mathrm{He}$, can be appreciated by their plots in Figure 4(b) where the normalized $j_{\exp }(t) / J_{\exp }$ permeation flux curves are presented.

In Figures 5(a)-5(d), we present the experimental $j_{\exp }(t) / J_{\exp }$ permeation curves obtained at temperatures between 295 and $350 \mathrm{~K}$ for $\mathrm{CO}_{2}, \mathrm{~N}_{2}, \mathrm{D}_{2}$, and He with $P_{\mathrm{HPS}}=$ $40 \mathrm{kPa}$ (symbols): the continuous lines are their fitting by the $j_{\text {norm }}(t)$ function. Note that the high signal-to-noise ratio of the present procedure permits to clearly distinguish the permeation curves obtained in the 295 to $350 \mathrm{~K}$ temperature range also using the small-size penetrants.

The Arrhenius plots of the permeability and diffusivity values are reported in Figures 6(a) and 6(b), respectively. Permeability values are reported in practical Barrer units

$$
1 \text { Barrer }=10^{-10} \frac{\mathrm{cm}^{3}(\mathrm{STP}) \mathrm{cm}}{\mathrm{cm}^{2} \mathrm{~s} \mathrm{cmHg}}=3.34 \times 10^{-18} \frac{\mathrm{mol} \mathrm{cm}}{\mathrm{cm}^{2} \mathrm{~s} \mathrm{~Pa}} \text {. }
$$

The activation energy value for permeation and the apparent value of the activation energy for diffusion were obtained fitting the permeability and diffusivity data by the following relations for the gas diffusivity $D$ and permeability $\Phi$ :

$$
\begin{aligned}
& D(T)=D_{0} e^{-\left(E_{D} / k_{B} T\right)}, \\
& \Phi(T)=\Phi_{0} e^{-\left(E_{\Phi} / k_{B} T\right)} .
\end{aligned}
$$

$E_{D}$ and $E_{\Phi}$ values of the examined test gases are reported in Table 2.

It is worthy to observe that the diffusivity values have been obtained by the analysis of the whole permeation curve which is composed, given the high sampling rate of the QMS, by a large ensemble of experimental data. This procedure thus allows more accurate evaluation of the gas diffusivity than using the value of a single parameter, as in the time lag method.

The obtained diffusivity and permeability values for $\mathrm{CO}_{2}$ as well as the pertinent activation energies are in well agreement with values previously obtained by the "constant-volume" approach [18] and with other literature data; see references therein. The same consideration holds for the permeability values and activation energy values for $\mathrm{H}_{2}$ and $\mathrm{N}_{2}$.

To conclude this discussion, it is worthy to comment the obtained results considering the physical-chemical properties of the employed test gases. Considering the diffusion data in Figure 6(b) and the penetrant properties in Table 1, we conclude that the penetrant diffusivity through the present LDPE samples decreases by increasing the molecular size. Moreover, comparing the permeability data in Figure 6(a) and the molecular physical-chemical properties in Table 1 , we conclude that the gas permeability of the present LDPE samples increases with the critical temperature of the test gas evidencing the important role of the gas condensation properties. In fact, $\mathrm{CO}_{2}$ and $\mathrm{N}_{2}$ have similar molecular size (see Table 1) and diffusivity (see Figure 6(b)) but the $\mathrm{CO}_{2}$ permeability is larger than that of $\mathrm{N}_{2}$. Note that 


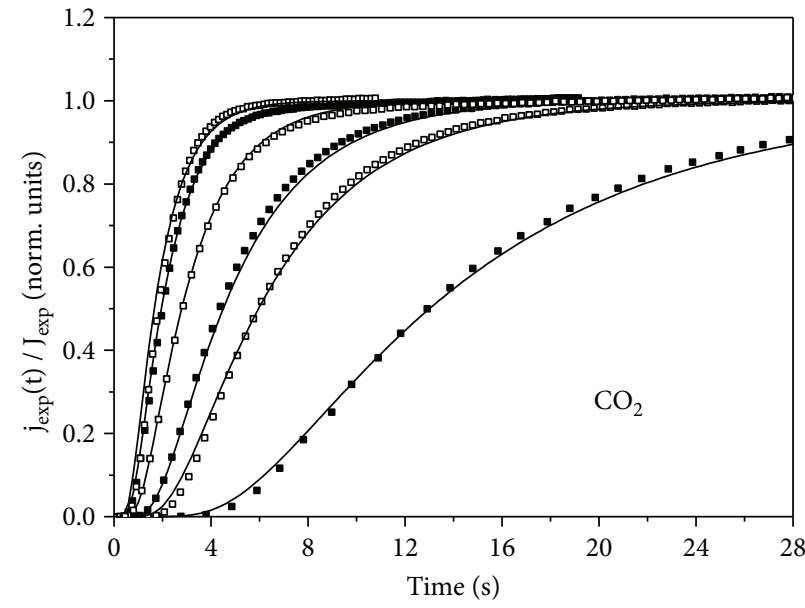

(a)

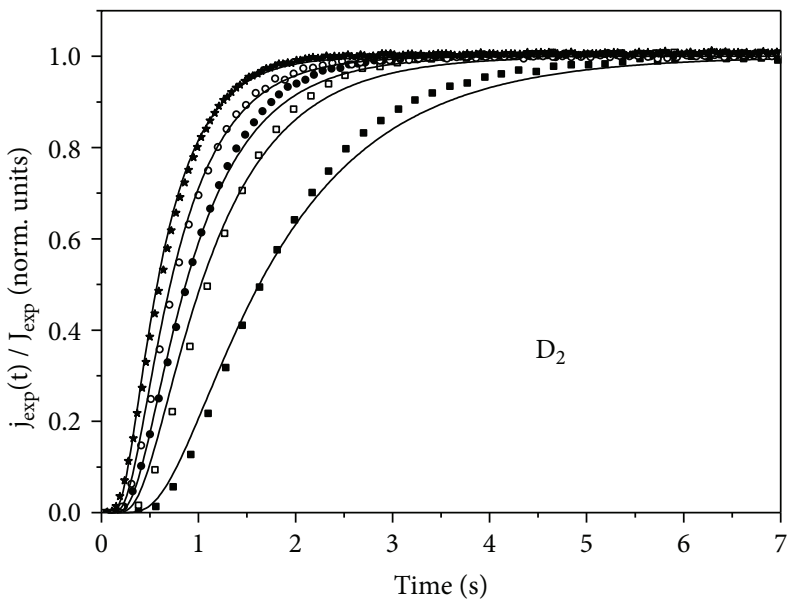

(c)

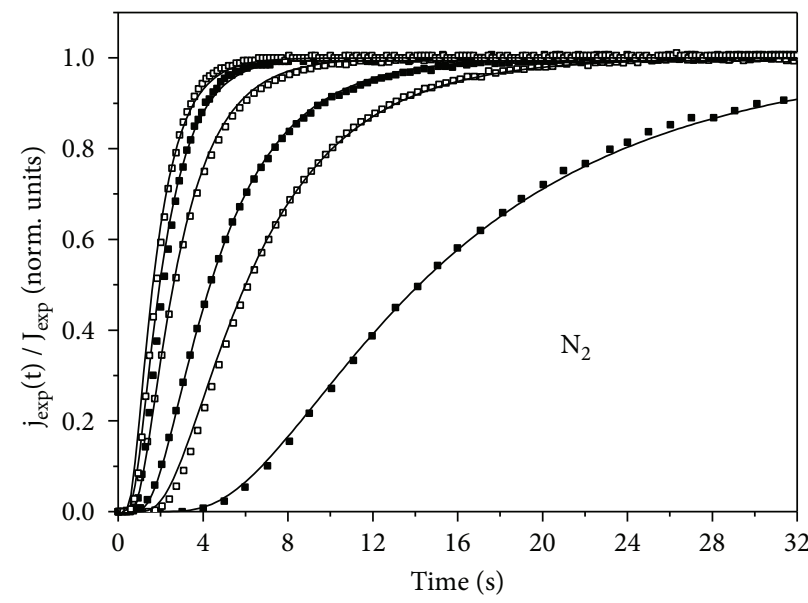

(b)

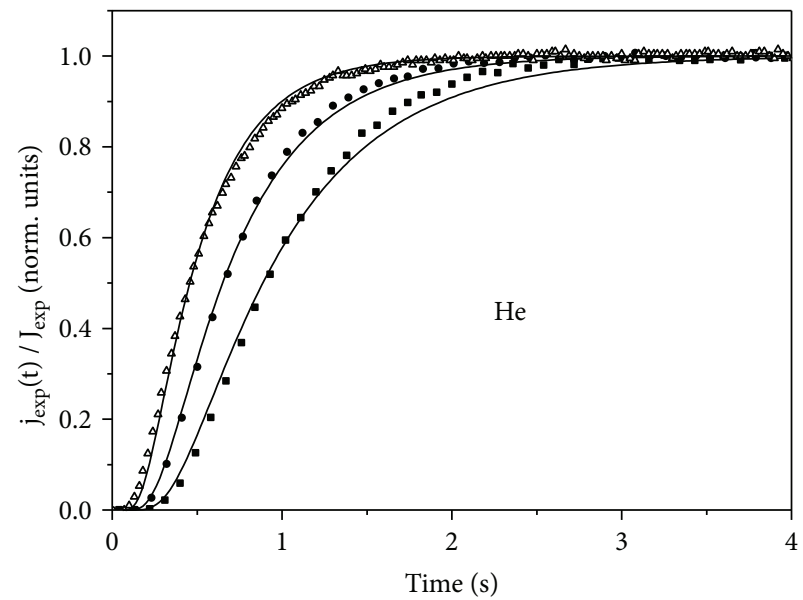

(d)

FIGURE 5: (a) Experimental $j_{\exp }(t) / J_{\exp }$ curves (symbols) pertinent to $\mathrm{CO}_{2}$ obtained at temperatures between $T=295 \mathrm{~K}$ and $T=351 \mathrm{~K}$ with $P_{\mathrm{HPS}}=40 \mathrm{kPa}$. Lines are fitting of the experimental curves; see text. (b) Experimental $j_{\exp }(t) / J_{\exp }$ curves (symbols) pertinent to $\mathrm{N}_{2}$ obtained at temperatures between $T=295 \mathrm{~K}$ and $T=351 \mathrm{~K}$ with $P_{\mathrm{HPS}}=40 \mathrm{kPa}$. Lines are fitting of the experimental curves; see text. (c) Experimental $j_{\exp }(t) / J_{\exp }$ curves (symbols) pertinent to $\mathrm{D}_{2}$ obtained at temperatures between $T=295 \mathrm{~K}$ and $T=345 \mathrm{~K}$ with $P_{\mathrm{HPS}}=40 \mathrm{kPa}$. Lines are fitting of the experimental curves; see text. (d) Experimental $j_{\exp }(t) / J_{\exp }$ curves (symbols) pertinent to He. To avoid confusion, in the figure, we only report curves obtained at 295,330 , and $351 \mathrm{~K}$ with $P_{\mathrm{HPS}}=40 \mathrm{kPa}$. Lines are fitting of the experimental curves; see text.

the same considerations hold also comparing the $\mathrm{H}_{2}$ and He transport parameters.

This scaling can be discussed considering the theoretical results presented in a recent paper of Dutta and Bhatia on the free volume structure and gas transport properties of polyethylene carried out by equilibrium molecular dynamic simulations [21]. The $\mathrm{CO}_{2}$ and $\mathrm{N}_{2}$ self-diffusivity values indicated by the authors are in the $10^{-6} \mathrm{~cm}^{2} / \mathrm{s}$ range, in accord with the present experimental evaluation. They also suggested that the temperature dependence of the penetrant self-diffusivity follows the Arrhenius behavior, as also observed in the present study. Simulations also revealed that the free volume of $\mathrm{PE}$ is composed by voids with size ranging from 1.5 to $3 \AA$. The larger $\mathrm{D}_{2}$ and He diffusivity compared with $\mathrm{CO}_{2}$ and $\mathrm{N}_{2}$ can be explained as consequence of the availability of diffusion sites with a diameter close to the $\mathrm{D}_{2}$ and He molecular sizes and thus is more efficient in accommodating these small-size penetrants.

\section{Conclusions}

We have described a procedure for the experimental study of gas permeation through polymer films. The procedure is based on the measure of the permeation signal in a continuously pumped analysis chamber by a calibrated quadrupole mass spectrometer. This procedure allows (i) very low detection limit of the permeation flux, $\sim 10^{-14} \mathrm{~mol} \cdot \mathrm{cm}^{-2} \cdot \mathrm{s}^{-1}$, and (ii) high signal-to-noise ratio in transient transport conditions also when studying polymer films having gas barrier properties. The improved performances permit to resolve the kinetics of the $\mathrm{He}$ and $\mathrm{D}_{2}$ transport processes, to obtain their diffusivity values up to temperatures close to those of 


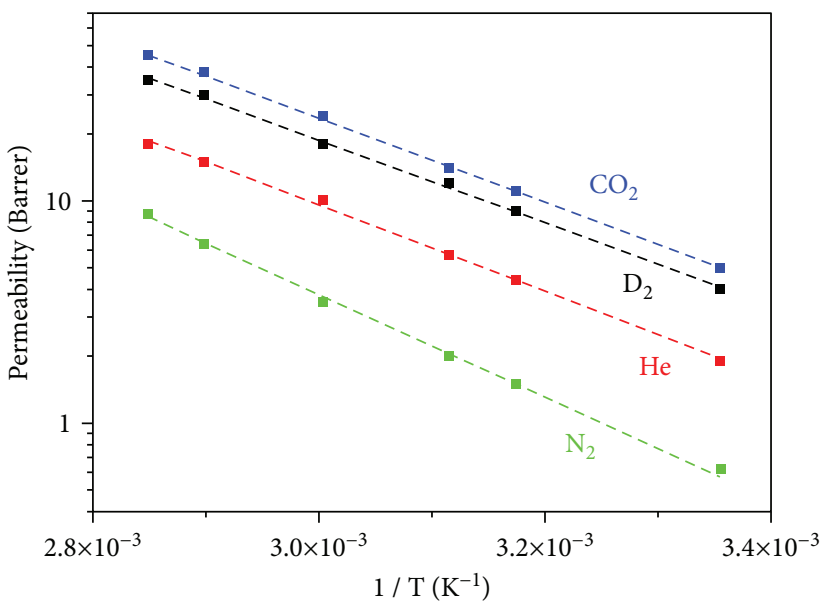

(a)

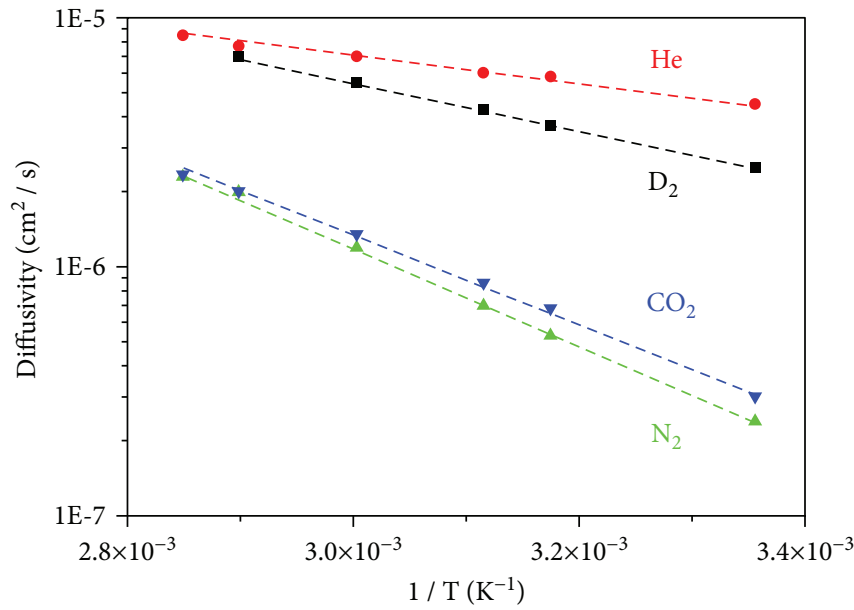

(b)

FIGURE 6: (a) Arrhenius plot of the gas permeability as obtained by analysis of the permeation flux value in stationary transport conditions. Permeability values are presented in practical units (Barrer). (b) Arrhenius plot of the gas diffusivity as obtained by the fitting of $j_{\exp }(t) / J_{\exp }$ curves; see Figures 5(a)-5(d).

TABLE 2: Activation energy for permeation $\mathrm{E}_{\Phi}$ and activation energy for diffusion $\mathrm{E}_{\mathrm{D}}$ as obtained by the Arrhenius plot of the permeability and diffusivity data in Figures 6(a) and 6(b).

\begin{tabular}{lcc}
\hline Test gas & $\mathrm{E}_{\Phi}(\mathrm{kJ} / \mathrm{mol})$ & $\mathrm{E}_{\mathrm{D}}(\mathrm{kJ} / \mathrm{mol})$ \\
\hline $\mathrm{CO}_{2}$ & $36.5 \pm 0.5$ & $34.0 \pm 0.3$ \\
$\mathrm{~N}_{2}$ & $43.2 \pm 0.8$ & $38.2 \pm 0.5$ \\
$\mathrm{D}_{2}$ & $33.2 \pm 0.8$ & $18.8 \pm 0.4$ \\
$\mathrm{He}$ & $37.2 \pm 0.7$ & $10.0 \pm 0.4$ \\
\hline
\end{tabular}

the LDPE melting and the pertinent activation energies of diffusion. This procedure can be applied for the study of the gas transport processes with the recently developed polymer materials for packaging applications to be applied in fields such as OLED technology. It is also of interest for the community of polymer scientists interested to the study of the correlation between the polymer-free volume structure and transport properties.

\section{Data Availability}

Experimental data supporting the described results can be obtained upon request to the corresponding author.

\section{Conflicts of Interest}

The author declares that there is no conflict of interests regarding the publication of this paper.

\section{Acknowledgments}

The help of M. Zanolli in the gas transport experiments is gratefully acknowledged.

\section{References}

[1] S. Matteucci, Y. Yampolskii, B. D. Freeman, and I. Pinneau, Materials Science of Membranes for Gas and Vapor Separation, John Wiley and Sons, Chichester, 2006.

[2] A. Lopez-Rubio, E. Almenar, P. Hernandez-Munoz, R. Catala, and R. Gavara, "Overview of active polymer-based packaging technologies for food applications," Food Reviews International, vol. 20, no. 4, pp. 357-387, 2004.

[3] J. S. Vrentas and J. M. Vrentas, "Evaluation of the free volume theory of diffusion," Journal of Polymer Science Part B: Polymer Physics, vol. 41, no. 5, pp. 501-507, 2003.

[4] H. Fujiita, "Notes on free volume theories," Polymer Journal, vol. 23, no. 12, pp. 1499-1506, 1991.

[5] R. P. White and J. E. G. Lipson, "Polymer free volume and its connection to the glass transition," Macromolecules, vol. 49, no. 11, pp. 3987-4007, 2016.

[6] P. N. Patil, D. Roilo, R. S. Brusa, A. Miotello, and R. Checchetto, "Influence of nano-level molecular packing on the gas transport properties in amine-modified epoxy resins," Polymer, vol. 58, pp. 130-138, 2015.

[7] P. N. Patil, D. Roilo, R. S. Brusa et al., "Free volumes and gas transport in polymers: amine-modified epoxy resins as a case study," Physical Chemistry Chemical Physics, vol. 18, no. 5, pp. 3817-3824, 2016.

[8] D. Roilo, P. N. Patil, R. S. Brusa et al., "Polymer rigidification in graphene based nanocomposites: gas barrier effects and free volume reduction," Polymer, vol. 121, pp. 17-25, 2017.

[9] D. Roilo, C. A. Maestri, M. Scarpa et al., "Cellulose nanofibrils films: molecular diffusion through elongated nanocavities," Journal of Physical Chemistry C, vol. 121, no. 28, pp. 1543715447, 2017.

[10] B. Flaconnèche, J. Martin, and M. H. Klopffer, "Transport properties of gases in polymers: experimental methods," Oil \& Gas Science and Technology, vol. 56, no. 3, pp. 245-259, 2001.

[11] R. M. Felder, "Estimation of gas transport coefficients from differential permeation, integral permeation, and sorption rate 
data," Journal of Membrane Science, vol. 3, no. 1, pp. 15-27, 1978.

[12] J. Crank, The Mathematics of Diffusion, Claredon, Oxford, 2nd edition, 1999.

[13] W. H. Lin and T. S. Chung, "Gas permeability, diffusivity, solubility, and aging characteristic of 6FDA-durene polyimide membranes," Journal of Membrane Science, vol. 186, no. 2, pp. 183-193, 2011.

[14] J. Ahn, W. J. Chung, I. Pinneau, and M. D. Guiver, "Polysulfone/silica nanoparticle mixed-matrix membranes for gas separation," Journal of Membrane Science, vol. 314, no. 1-2, pp. 123-133, 2008.

[15] J. P. G. Villaluenga and B. Seoane, "Experimental estimation of gas-transport properties of linear low-density polyethylene membranes by an integral permeation method," Journal of Applied Polymer Science, vol. 82, no. 12, pp. 3013-3021, 2001.

[16] W. J. Koros, A. H. Chan, and D. R. Paul, "Sorption and transport of various gases in polycarbonate," Journal of Membrane Science, vol. 2, pp. 165-190, 1977.

[17] K. Marsch and B. Bugusu, "Food packaging roles, materials and environmental issues," Journal of Food Science, vol. 72, no. 3, pp. R39-R55, 2007.

[18] R. Checchetto, A. Miotello, L. Nicolais, and G. Carotenuto, "Gas transport through nanocomposite membrane composed by polyethylene with dispersed graphite nanoplatelets," Journal of Membrane Science, vol. 463, pp. 196-204, 2014.

[19] P. A. Redhead, J. P. Hobson, and E. V. Kornelsen, The Physical Basis of Ultrahigh Vacuum, American Institute of Physics, New York, NY, USA, 1993.

[20] J. S. Lewis and M. S. Weaver, "Thin-film permeation-barrier technology for flexible organic light-emitting devices," IEEE Journal of Selected Topics in Quantum Electronics, vol. 10, no. 1, pp. $45-57,2004$.

[21] R. C. Dutta and S. K. Bhatia, "Transport diffusion of light gases in polyethylene using atomistic simulations," Langmuir, vol. 33, no. 4, pp. 936-946, 2017. 


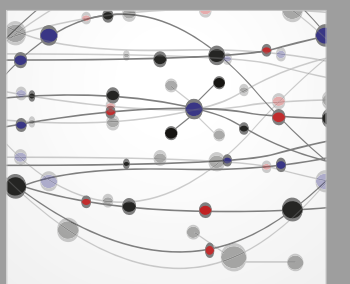

The Scientific World Journal
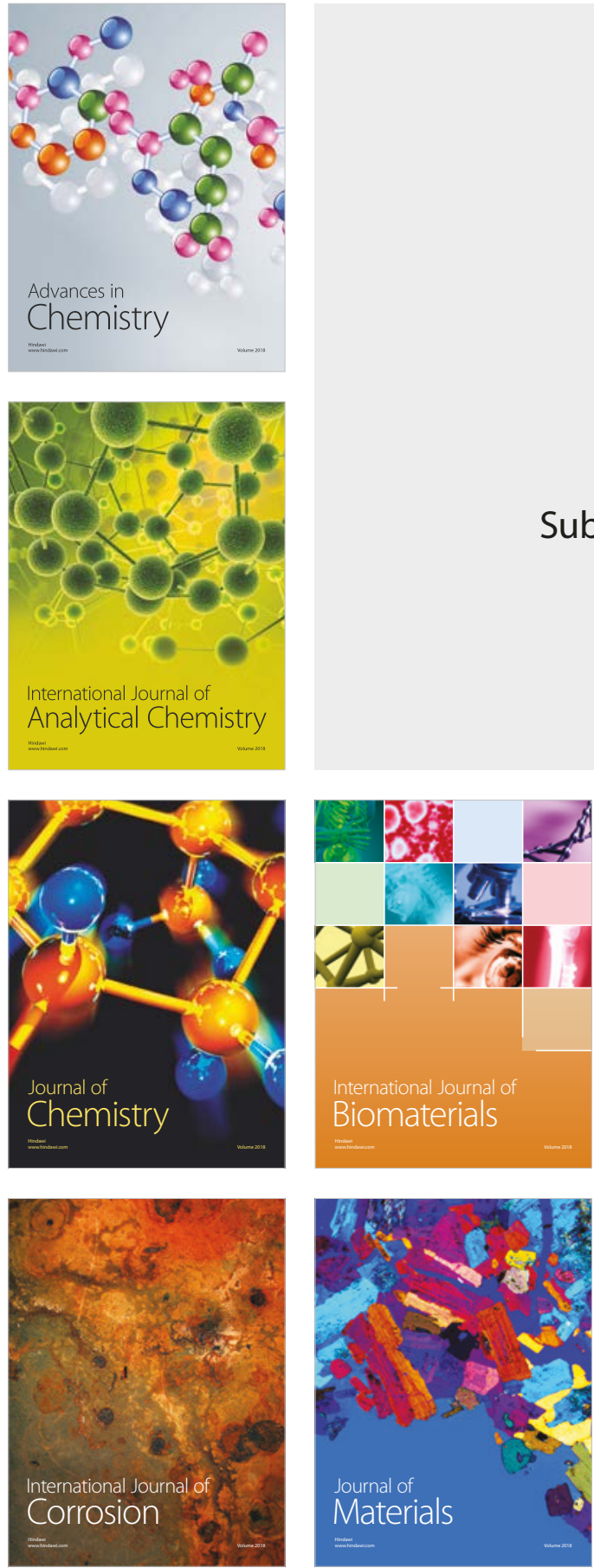

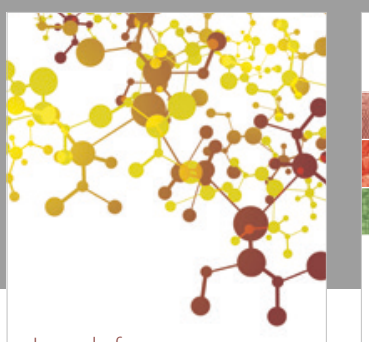

Journal of

Applied Chemistry
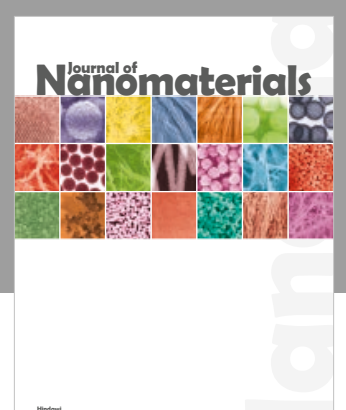

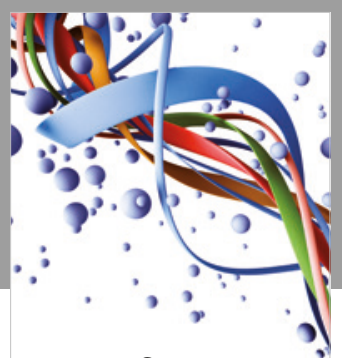

Scientifica

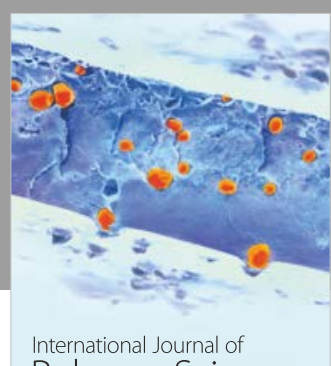

Polymer Science

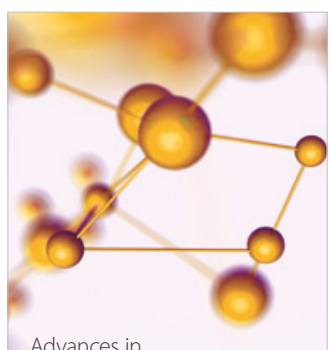

Physical Chemistry
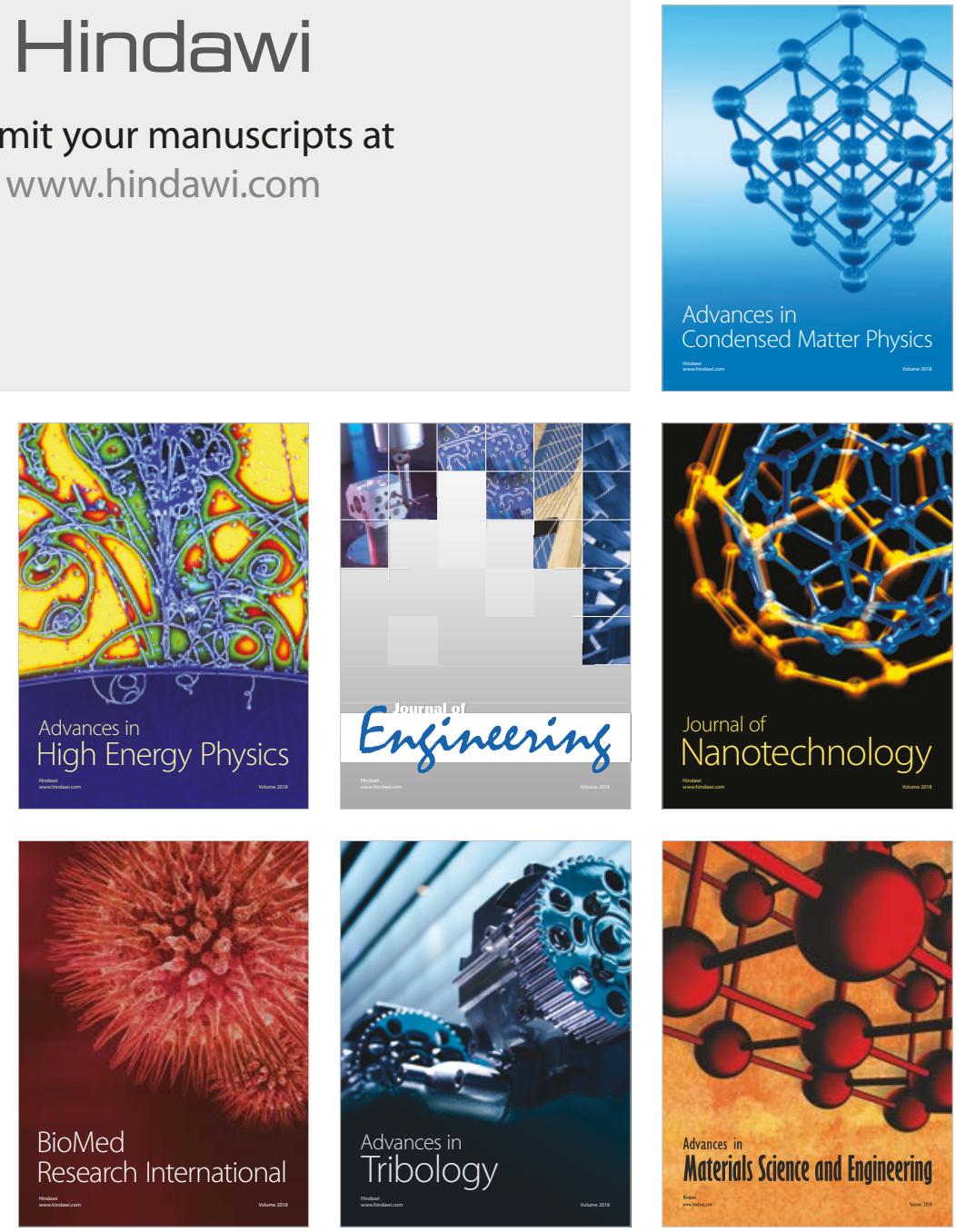